\title{
Scratching, hyperkeratosis and ectoparasites in a guinea pig
}

Wieland Beck, DVM, DEVPC 1 , Florian Buck, $D V M^{2}$ \& Nikola Pantchev, DVM ${ }^{3}$

Small mammals once used frequently as laboratory animals have become increasingly popular as pets in Germany. The number of small mammal patients being presented to companion animal veterinarians has also increased continuously over the past years. The most common afflictions in these pets are skin diseases.

One 3-year-old male guinea pig weighing $400 \mathrm{~g}$ was brought to the Small Animal Practice in Rottenburg, Germany. The owners fed the animal a mixed diet of fresh vegetables and guinea pig pellets and supplied water ad libitum from a water bottle that they changed regularly. The owners described a history of extreme restlessness and intense scratching behind both ears in the guinea pig. On clinical examination, the guinea pig was bright, alert and responsive. It was mildly emaciated, and its coat was unkempt. Most noticeable were widespread patches of flaky, thickened skin (Fig. 1). Alopecia was prominent around its eyes and several areas of bleeding

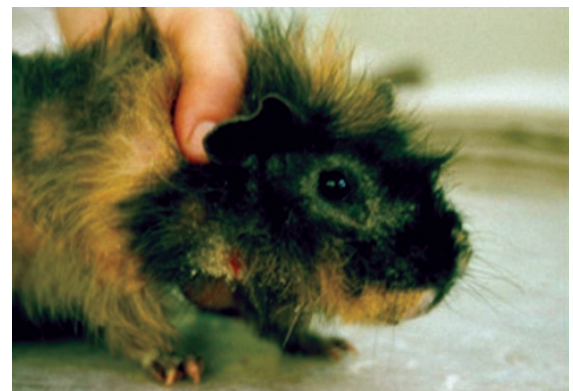

FIGURE 1 | Flaky, thickened, pruritic skin lesions in a 3-year-old male guinea pig that presented for agitation and itching.

raw skin were seen on its head and neck (Fig. 1). The guinea pig exhibited severe pruritus. The owners reported that the animal scratched constantly and had experienced sporadic epileptiform seizures. The clinical veterinarian scraped sections of the scaly skin and examined the epidermal debris microscopically. $\mathrm{He}$ saw numerous eggs and arthropods with four pairs of legs (Fig. 2).

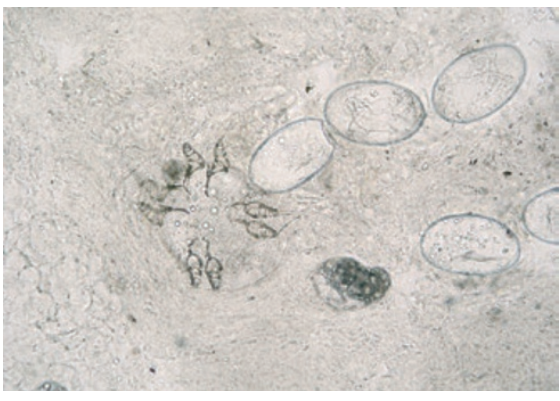

FIGURE 2 | Ventral view of an arthropod ectoparasite and numerous eggs seen microscopically in the epidermal debris (mediumpower magnification).

Based on the clinical history, the appearance of the guinea pig and the microscopic examination of the epidermal scrapings, what is the arthropod seen? What is the common name of this skin condition? How would you treat it? Is it zoonotic?

What's your diagnosis?

\footnotetext{
${ }^{1}$ Institute for Comparative Tropical Medicine and Parasitology, Ludwig-Maximilians-University Munich, Leopoldstrasse 27, D-80802 Munich, Germany. ${ }^{2}$ Small Animal Practice, Landshuter Strasse 23, D-84056 Rottenburg, Germany. ${ }^{3}$ Vet Med Labor GmbH, Division of IDEXX Laboratories, Postfach 1110 , D-71611 Ludwigsburg, Germany. Correspondence should be addressed to W.B. (Wieland.Beck@tropa.vetmed.uni-muenchen.de).
} 